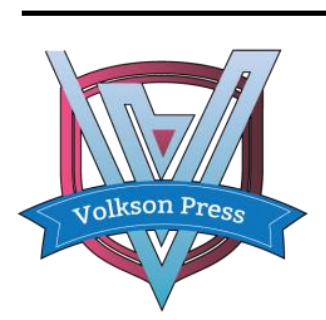

\title{
PREPARATION AND PROPERTIES OF SELF-EMULSIFIED CATIONIC WATERBORNE EPOXY RESIN MODIFIED SIZING AGENT
}

\author{
Xu Feng, Jianhua Li, Hongfei Jia, Zhenyu Wang, Chuncai Yang* \\ Jilin Institute of Chemical Technology Chengde Street, Jilin, China. \\ *Corresponding Author Email: 2941883707@qq.com
}

This is an open access article distributed under the Creative Commons Attribution License, which permits unrestricted use, distribution, and reproduction in any medium, provided the original work is properly cited

\section{ARTICLE DETAILS}

\section{Article History:}

eceived 26 June 2018

Accepted 2 July 2018

Available online 1 August 2018

\section{ABSTRACT}

This article reports a preparation and properties of self-emulsified cationic waterborne epoxy modified resin as a film former of sizing agent. There were two major steps to prepare cationic waterborne epoxy modified emulsion polymers. The first step was to synthesize a linear polymer containing tertiary amine in its main chain by the reaction of epoxy resin with hydrophobic primary aliphatic/aromatic amine and hydrophilic polyetheramine. The second step was to neutralize the polymer synthesized in the first step by acetic acid to produce a cationic polymer and then dispersed it into water to produce cationic waterborne emulsion polymer. A series of parallel experiments of synthetic reaction between epoxy resin and mixture of two kinds of primary amine were completed and the optimal reaction temperature and time were concluded as $110^{\circ} \mathrm{C}$ and 3 hours respectively. The amphiphilic properties of cationic waterborne epoxy modified emulsion polymers were adjusted by the ratio of the primary aliphatic/aromatic amine with polyetheramine. The particle size of epoxy modified emulsion polymers prepared in this paper was small and controllable. The emulsion stability was good and was stored at room temperature for more than three months and there was no precipitation and phase separation.

\section{KEYWORDS}

Self-emulsified, cationic, waterborne, epoxy resin, sizing agent, film former.

\section{INTRODUCTION}

Carbon fiber reinforced composite material (CFC) was an advanced composite material with high specific strength, high specific modulus and structural design [1]. It was widely concerned and used in aerospace, automotive manufacturing, medical style appliances and other fields. However, carbon fiber was brittle and had low elongation and chemical inertness on the surface, the strength of carbon fibers was reduced due to mechanical friction which was easy to produce split filament and monofilament fracture during production and processing of CFC [2]. The structure of the carbon fiber determined the properties of the carbon fiber, which in turn determined the properties of CFC. Sizing agent was a key optimization process in the production of carbon fibers $[3,4]$. The sizing agent formed a layer of nanometer-scale polymer film on the surface of the carbon fiber, and also formed an interfacial layer between the fiber body and the resin matrix, and functions as an adhesive. On the one hand. The sizing agent could increase the bundling property of the fibers, aggregate carbon fibers together, and improve the process performance. On the other hand, by introducing polar chemical functional groups on the surface of carbon fibers that increased surface activity and the chemical bonding between the fibers and the resin. At present, the major resin used as matrix in $\mathrm{CFC}$ is roughly classified into unsaturated polyester, epoxy, polyurethane, acrylic and so on, therefore various kinds of sizing agent corresponding to the resin would be required so that the resin have the strongest chemical bonding and Van der Waals forces to the sized fiber [5]. In recent years, as people's awareness of environmental protection continues to increase, the volatile solvent-based epoxy resins used as sizing agent are gradually being replaced, and waterborne epoxy sizing agents are increasingly sought after by people [6]. According to the synthesized technologies of waterborne epoxy emulsion reported in the reference, there were chemical modification, physical ball milling and phase inversion emulsification methods [7]. Bisphenol An epoxy resin is a kind of hydrophobic and water-insoluble chemical and hard to disperse into water, so large amounts of surfactant and high-speed agitation are needed to emulsify epoxy resin. However, the epoxy emulsion obtained by adding emulsifier and high-speed agitation has uneven particle size distribution, poor storage stability and inefficient performance of the composite $[8,9]$. In order to prepare a water-based epoxy emulsion with good stability, it is necessary to modify epoxy resin chemically to become hydrophilic. Therefore, this article will mainly report how to modify epoxy resin chemically into amphiphilic polymer, to change epoxy modified emulsion polymer structure, to study its properties and to optimize the formulation of sizing agent to be fit for the process of production and processing of both hydrophobic carbon fiber and hydrophilic basalt fiber or glass fiber [10-12].

\section{EXPERIMENTAL PART}

\subsection{Experimental Materials and Instruments}

Epoxy resin E-1NT (Jilin Qianren); Octadecylamine (Tianjin Fuchen Chemical); $\mathrm{HCl}, \mathrm{KOH}$, Acetone (Tianjin Dongfang Chemical); deionized water (Laboratory homemade); Analytical Balances (Tianjin Tianma); Instant heated water bath, (Gongyi Yuhua); Blender (Changzhou Aohua); High-speed centrifuge(Anhui Zhongke Zhongjia); Infrared spectrometer(Frontier US PerkinElmer Corporation); ZetaPlus laser particle size analyzer(Brookhaven Instruments, USA).

\subsection{Experimental Method}

The mixture of certain mole ration of hydrophilic polyetheramine (MW 2000) and primary aliphatic/aromatic amine such as octadecylamine with hydrophobic groups was dissolved by acetone. The Bisphenol A epoxy resin (185 equivalent) and the aforementioned mixture of amine are added at a room temperature to a reaction flask equipped with heating oil bath mechanical stirrer、distillation head with condenser jacket on side 
arm 、 and distillation receiver. The initial reaction temperature was set to $40{ }^{\circ} \mathrm{C}$ under nitrogen atmosphere. After the reaction temperature was stabilized at $40^{\circ} \mathrm{C}$ for 10 minutes, the temperature of heating oil bath was set to $70{ }^{\circ} \mathrm{C}$. the reaction temperature was gradually increasing with acetone distilling out. The final reaction was set at $110{ }^{\circ} \mathrm{C}$ for three hours. After the reaction temperature was cooled down to $40{ }^{\circ} \mathrm{C}$, a calculated amount of glacial acetic acid was added to neutralize the epoxy modified polymer and then a certain amount of water was poured to the glass flask and self-emulsified cationic waterborne epoxy modified polymer had been prepared.

\subsection{Performance Testing}

The characterization of epoxy modified polymer was taken by using a Fourier infrared spectrometer. The particle size of the self-emulsified emulsion polymer were determined by laser particle size scattering instrument. The emulsion stability was investigated by centrifuge and long term of storage.

\section{RESULTS AND DISCUSSION}

\subsection{Hydrophilic and hydrophobic properties}

The mole ratio of n-octadecylamine (SA) with polyetheramine (M1) was designed as $0.8: 0.2 ; 0.6: 0.4,0.5: 0.5,0.4: 0.6$ and 0.2:0.8, the reaction between Bisphenol A epoxy resin and the mixture of SA and M1 took place to produce linear polymer containing tertiary amine in its main chain. The reaction temperature was set to $110^{\circ} \mathrm{C}$ for three hours. After the temperature was brought back to room temperature, glacial acetic acid was added as a neutralizing agent to generate cationic polymers and finally $60 \sim 70 \%$ deionized water was added to the cationic polymers for self-emulsification. The resulting emulsions are marked as R1, R2, R3, R4 and R5 respectively for analyzing particle size. The particle size of cationic waterborne emulsion polymer is becoming smaller obviously as mole ratio of SA and M1 decreasing as shown in Figure 2. The testing result confirms that the particle size of emulsion can be controlled by the design of polymer structure, the more hydrophilic segment and the smaller particle size of emulsion.

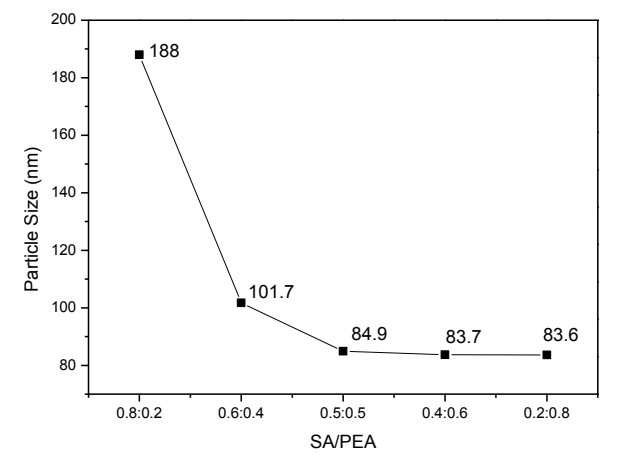

Figure 1: Relationship between the particle size of emulsion and proportion of hydrophilic and hydrophobic segment

\subsection{FT-IR spectra}

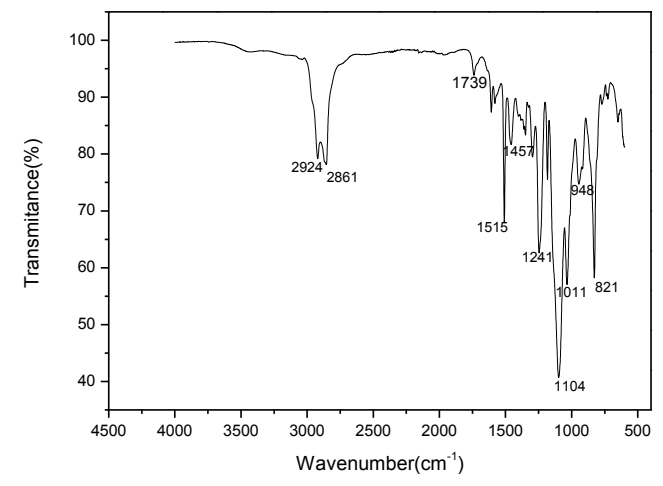

Figure 2: FT-IR spectra of epoxy modified polymer

Figure 2 shows the FT-IR spectral characterization of the epoxy modified polymer, R3 chosen as a representative product. Peaks near $2924 \mathrm{~cm}^{-1}$ and $2861 \mathrm{~cm}^{-1}$ are considered to be the stretching vibration peaks of $-\mathrm{CH}_{3}$ and $\mathrm{CH}_{2}$. The peaks at both $821 \mathrm{~cm}^{-1}$ and $948 \mathrm{~cm}^{-1}$ are assigned to the characteristic absorption peaks of epoxy groups that confirmed the epoxy modified polymer had been terminated by epoxy group. $1104 \mathrm{~cm}^{-1}$ is the absorption peak of C-O, and $1011 \mathrm{~cm}^{-1}$ is the absorption peak of C-N. Both the $1515 \mathrm{~cm}^{-1}$ and $1457 \mathrm{~cm}^{-1}$ are the characteristic absorption peaks of the benzene ring skeleton. No characteristic peak of $-\mathrm{NH}_{2}$ and $-\mathrm{NH}$ indicated that octadecylamine and polyetheramine had completed reaction with Bisphenol A epoxy resin. All above present or absent characteristic peaks proved that hydrophilic and hydrophobic segments are well grafted together and the cationic waterborne epoxy modified emulsion polymer as film former of sizing agent had been prepared successfully.

\subsection{Optimization of experimental conditions}
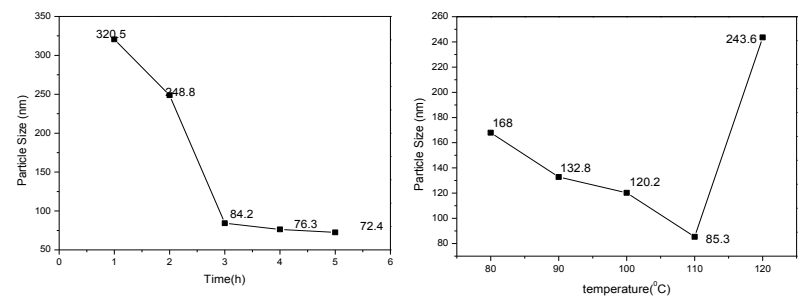

Figure 3: (a) effect of reaction time on particle size of epoxy modified emulsion polymer, (b) effect of reaction temperature on particle size of epoxy modified emulsion polymer

Figure 3(a) and Figure 3(b) show the effect of reaction temperature and time on particle size of epoxy modified emulsion polymer respectively. The particle size is decreasing as the reaction of Bisphenol A epoxy resin with SA and $\mathrm{M} 1$ is going on at $110^{\circ} \mathrm{C}$, when the reaction time reaches 3 hours, the particle size decreases to $84.2 \mathrm{~nm}$. After $3 \mathrm{hrs}$ reaction, the particle size was in range of $70 \mathrm{~nm}$ to $80 \mathrm{~nm}$ and the decrease of particle size was not obvious, therefore the optimal reaction time was considered to be three hours. As shown in figure 3(b) the particle size is quickly decreasing with reaction temperature rising, however when the reaction temperature exceeds $110^{\circ} \mathrm{C}$, the particle size is increasing, which indicated the epoxy reacting with the -OH group and resulting in an partial crosslinking reaction, therefore the optimal reaction temperature is about $110^{\circ} \mathrm{C}$.

\subsection{Emulsion Storage Stability}

As shown in Figure 4, 30ml of epoxy modified emulsion polymer from sample R1 to R5 were stored at room temperature to watch if and when the precipitation or phase separation occurs. Figure 4(a), (b), (c) and (d) stands for storage of 2 weeks, 1 month, 2 months and 3 months respectively. All emulsion samples has no precipitation and phase separation within 3 months storage, indicating that the emulsion had good stability.

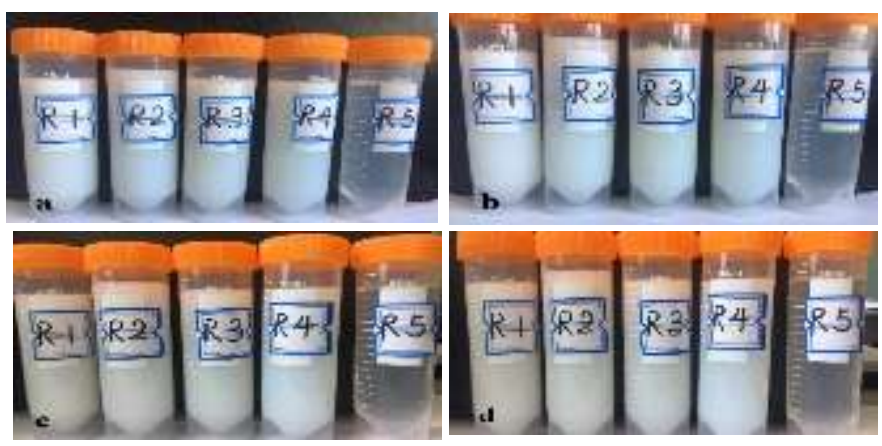

Figure 4: Emulsion stability at room temperature (a)Resting for 2 weeks; (b) Resting for 1 month; (c) Resting for 2 months; (d) Resting for 3 months

\subsection{Emulsion stability of centrifugation test}

As shown in Table $1,30 \mathrm{ml}$ of epoxy modified emulsion polymer from sample R1 to R5 were centrifuged at $2000 \mathrm{r} / \mathrm{min}$ for 3 hours and checked every half hour. All samples of emulsion from R1 to R5 had no sediment and phase separation, indicating that emulsion stability was good. 
Table 1: Emulsion stability of centrifuge test

\begin{tabular}{|c|c|c|c|c|c|c|c|}
\hline & $0.5 \mathrm{~h}$ & $1 \mathrm{~h}$ & $1.5 \mathrm{~h}$ & $2.5 \mathrm{~h}$ & $3 \mathrm{~h}$ & & \\
\hline $\mathrm{R} 1$ & & $\mathrm{nc}$ & layered & non-layered & non-layered non-layered & non-layered & non-layered \\
\hline $\mathrm{R} 2$ & & & layered & non-layered & non-layered non-layered & non-layered & non-layered \\
\hline R3 & & & layered & non-layered & non-layered non-layered & non-layered & non-layered \\
\hline $\mathrm{R} 4$ & & & layered & non-layered & non-layered non-layered & non-layered & non-layered \\
\hline R5 & & & layered & non-layered & non-layered non-layered & non-layered & non-layered \\
\hline
\end{tabular}

\section{CONCLUSION}

A series of self-emulsified and particle size controllable cationic waterborne epoxy modified emulsion polymer had been prepared based on reaction of epoxy resin with primary amine. The optimal reaction temperature and time between Bisphenol A epoxy resin with mixture of octadecylamine and polyetheramine was $110^{\circ} \mathrm{C}$ and 3 hours. The particle size of epoxy modified emulsion polymer is decreasing with the ratio of hydrophilic polyetheramine in the mixture increasing, which supports hydrophilic segment in favor of the self-assemble of small size micelles. The emulsion stability is good according to testing of both long term of storage and high-speed centrifugation of the epoxy modified emulsion polymer. Other kinds of primary aliphatic/aromatic amine such as hexamines, laurylamine, aniline or naphthylamine had replaced octadecylamine to produce a similar kind of epoxy modified emulsion polymer, which will tailor the hydrophilic or hydrophobic properties of the emulsion, for example carbon fiber prefers relatively more hydrophobic emulsion polymer as a film former of sizing agent, and glass fiber likes more hydrophilic emulsion polymer as a film former of sizing agent.

\section{REFERENCES}

[1] Ruliang, Z., Yudong, H., Li, L., Dan, S. 2011. Effect of Molecular Weight of Sizing Agent on the Apparent Properties and Interfacial Properties of Carbon Fiber [J]. Material Science and Technology, 19 (3), 137-143.

[2] Yang, Y., Lu, C.X., Su, X.L., Wang, X.K. 2007. Effects of Emulsion Sizing with nano-SiO2 on Interfacial Properties of Carbon Fiber/Epoxy Composites [J]. Journal of Materials Science, 42, 6347-52.

[3] Soutis, C. 2005. Fibre reinforced composites in aircraft construction [J]. Progress in Aerospace Sciences, 41 (2), 143-151.

[4] Yan, Z., Zhenzhong, Y., Delu, Z. 2000. Influence of Hydrophilic Component Content of Emulsifier Molecules on Phase Inversion Emulsification Process [J]. Journal of Chemistry in Colleges and Universities, (02), 327-329.

[5] Xiang, K.K.C., Lei, S.X., Bismarck, A. 2007. Fluorination of carbon fiber surface and epoxy resins [J]. Carbon, 45 (4), 775-784.

[6] Ying, Z.Z., Hui, H.Y., Bing, L. 2004. Studies of water bone emulsion of chemically modified epoxy resin [J]. Polymer Advanced Technology, 15, 26-29.

[7] Qin, F.C., Lin, W.J., Tao, Z. 2014. Interlaminar improvement of carbon fiber/epoxy composites via depositing mixture of carbon nanotubes and sizing agent [J]. Applied Surface Science, 321, 1-9.

[8] Lei, Q., Yangtao, L., Meng, G., Gangyi, Z. 2016. Research Progress of Surface Treatment and Sizing Agent for Carbon Fiber [J]. Fiber Composite, (1), 33-35.

[9] Haojie, Y., Shouchun, Z., Chunxiang, L., Haojie, F. 2014. Research Progress in Effect of Sizing Agent on Interfacial Bonding of Carbon Fiber Reinforced Composites [J]. New Chemical Materials, (10), 1-3.

[10] Yang, L., Yuefang, W., Yonggang, Y. 2009. Effect of Epoxy Sizing Agent on Properties of PAN Based Carbon Fiber [J]. Synthetic Fiber Industry, 32 (2), 1-3.

[11] Chao, L., Lamei, G. 2011. Preparation and Performance Analysis of Waterborne Epoxy Resin Carbon Fiber Sizing Agent [J]. Industrial Textiles, (6), 40-42.

[12] Xingyao, W., Meng, L., Yaofeng, Z. 2016. Effect of Water Based Sizing Agent on Interfacial Properties of Carbon Fiber Surface and Carbon Fiber/Epoxy Resin Composite [J]. Journal of Composite Materials, 33 (2), 273-279.

\section{ABOUT THE AUTHORS}

Xu feng (1993-) male, graduate student, The main research direction is epoxy sizing agent.

Chuncai Yang(1968-)male, Thousand Talents Program Specifically-Invited Expert. 